\title{
Índice de contribución ecológica: Un método simple y confiable para determinar la condición de pastizales
}

\section{Ecological contribution index: A simple and reliable method to estimate range condition}

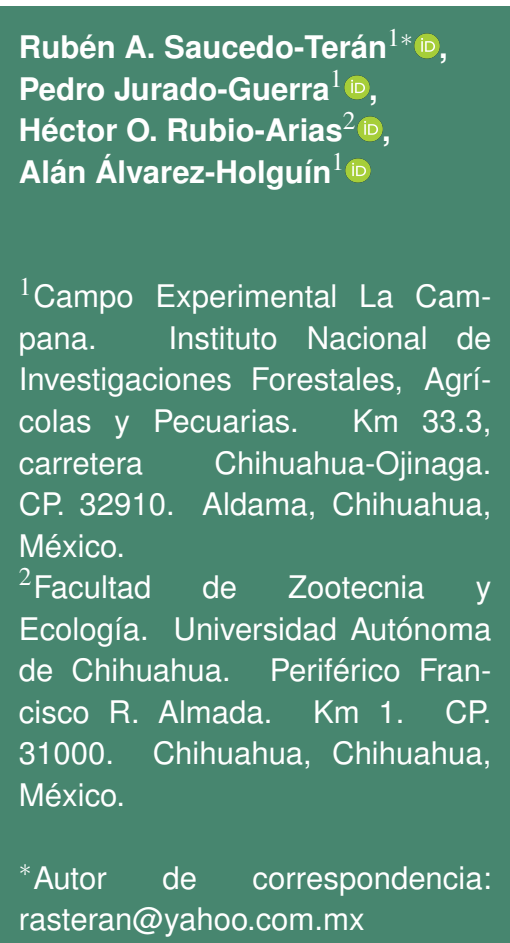

Artículo científico

Recibido: 08 de octubre 2020

Aceptado: 22 de abril 2021

Como citar: Saucedo-Terán RA, Jurado-Guerra P, Rubio-Arias $\mathrm{HO}$ Álvarez-Holguín A (2021) Índice de contribución ecológica: Un método simple y confiable para determinar la condición de pastizales. Ecosistemas y Recursos Agropecuarios 8(1): e2732. DOI: 10.19136/era.a8n1.2732
RESUMEN. Las herramientas de evaluación de pastizales presentan limitantes que las hacen difíciles de utilizar. Por lo anterior, el objetivo del presente trabajo fue proponer y describir una metodología sencilla y confiable para evaluar pastizales. La propuesta consiste en la elaboración de un índice de contribución ecológica del pastizal (ICEP) a través de la estimación de cuatro indicadores. Se estudiaron 29 ranchos ganaderos, en los que se determinó la condición de los pastizales y el ICEP. La condición de los pastizales se calculó con la metodología de COTECOCA. El ICEP se obtuvo por estimaciones visuales de la cobertura general de vegetación, cobertura de especies forrajeras perennes, capacidad de retención de agua y estabilidad de suelos. Los datos obtenidos se sometieron a pruebas de Chi cuadrada, análisis de correspondencias y regresión logística para determinar su confiabilidad. No se obtuvieron evidencias estadísticas que sustenten la independencia entre los datos generados mediante el método propuesto y los del método tradicional $(p>$ 0.05 , Chi cuadrada de Pearson) en los tres tipos de vegetación estudiados. De igual manera, los análisis de correspondencias y de regresión logística ordinal (gama de Goodman-Kruskal $=0.91, p<0.01$ ) mostraron que existe una relación estrecha entre los resultados del ICEP y los de la condición del pastizal.

Palabras clave: Cobertura de vegetación, especies forrajeras perennes, estabilidad de suelos, retención de agua.

ABSTRACT. Current rangeland assessment techniques show constraints making them difficult to use. Our objective was to propose and describe a reliable and simple method to evaluate rangelands. The method consists of a rangeland ecological contribution index (RECl) through estimation of four indicators. Range condition and $\mathrm{RECl}$ were estimated at 29 beef cattle ranches. Range condition was calculated by COTECOCA (Climax) methodology. RECl was estimated through visual observations of total vegetation cover, perennial forage species cover, water retention capacity, and soil stability. Data collected were analyzed by Chi-Square test, correspondence analysis, and logistic regression to determine their reliability. No statistical evidences ( $p<0.01$; Chi-Square) were found that support independence between $\mathrm{RECl}$ and range condition data at all vegetation types. Also, correspondence analysis and ordinal logistic regression (Goodman-Kruskal's gamma $=0.91, p<0.01$ ) showed a close relationship between results of $\mathrm{RECl}$ and range condition.

Key words: Plant cover, perennial forage species, soil stability, water retention. 


\section{INTRODUCCIÓN}

La evaluación de los pastizales es una tarea de gran importancia, debido a que es la base para el diseño de planes de manejo enfocados hacia la interrupción o mitigación de los procesos de deterioro y, en su caso, la estabilización o mejoramiento de su estado de salud. Bajo ese contexto, se han generado diversos métodos e indicadores de evaluación de este importante ecosistema. Al respecto, Dyksterhuis (1949) desarrolló un sistema cuantitativo que consiste en comparar la cubierta vegetal actual contra una cubierta vegetal clímax. Dado que en la vegetación clímax sólo son incluidas las plantas de importancia forrajera, algunas plantas de bajo valor forrajero son consideradas como vegetación menos deseable o indeseable, a pesar de cubrir otras funciones importantes desde el punto de vista ecológico (Karlin et al. 2013). Expertos del Consejo Nacional de Investigación de Estados Unidos sugirieron que en la determinación de la condición de los pastizales se deben incluir otros factores, adicionales a la comunidad clímax (NRC 1994). Bajo ese enfoque, surgió el concepto de salud del pastizal el cual se define como el grado en que la integridad del suelo, vegetación, agua y los procesos ecológicos están balanceados y son sostenibles (USDA-NRCS 2003). Mientras que Van der Westhuizen et al. (2005) desarrollaron una técnica basada en la asignación de valores ecológicos a las especies presentes con respecto a un gradiente de degradación, con las principales ventajas de que es una técnica simple y fácil de usar. Otra alternativa es el método conocido como estado de salud, el cual se basa en 17 indicadores con los que se califican la estabilidad del suelo, la funcionalidad hidrológica y la integridad biótica del ecosistema (Pyke et al. 2002, Pellant et al. 2005). En tanto que Tongway y Hindley (2004) desarrollaron una técnica denominada análisis funcional de los ecosistemas, mediante la creación de índices basados en mediciones de campo sobre estabilidad de los ecosistemas, infiltración de agua y ciclo de nutrientes. Al repecto, Sinclair et al. (2015) crearon un modelo para cuantificar la condición de pastizales mediante 8 indi- cadores ecológicos, seleccionados en consenso por especialistas en pastizales, botánica, fauna silvestre y conservacionistas. Posteriormente, Sinclair et al. (2021) ampliaron el número de indicadores a 14 y validaron el modelo en diferentes tipos de pastizales con resultados favorables. No obstante, este modelo solo considera indicadores relacionados con la vegetación y no otros indicadores de estabilidad ecosistémica, como es la estabilidad del suelo. Mientras que Ahmadpour et al. (2016) introdujeron un nuevo método en el que la condición del pastizal se concibe como la tasa entre los beneficios actuales de un pastizal y los costos de operación para su rehabilitación ecológica. De acuerdo con los autores, la principal ventaja de su método es que la condición es estimada como un coeficiente en lugar de una clasificación categórica, evitando así que dos sitios de pastizales con puntuaciones parecidas sean catalogados en dos clases diferentes y viceversa. También se han generado diversos modelos para monitorear la salud de los pastizales basados en sistemas de información geográfica. Sin embargo, estos modelos tienen la limitante de que tienen baja resolución espacial, por lo que no pueden generar información precisa de sitios en específico (Shao et al. 2016, Han et al. 2018, Jones et al. 2018).

Las herramientas actuales de evaluación de los pastizales presentan algunas limitantes que las hacen imprácticas, se basan en conceptos teóricos que han perdido su actualidad, son complejas y de uso específico para casos particulares (Novák 2004, Mueller et al. 2014). Además, no dan respuesta sobre otras funciones importantes de los pastizales como son los servicios ambientales y la conservación de la biodiversidad (Fuhlendorf y Engle 2001, Ludwig y Bastin 2008, Maes et al. 2016). Por lo tanto, se hace necesario disponer de un método que, además de incluir factores ecológicos, sea confiable, simple y factible de ser usado por cualquier persona, desde investigadores especializados en el tema, hasta agentes de cambio y ganaderos. El objetivo del presente trabajo fue proponer, describir y evaluar una nueva metodología para la evaluación de pastizales. 


\section{MATERIALES Y MÉTODOS}

El trabajo se llevó a cabo en pastizales medianos, pastizales amacollados arborescentes y matorrales, que son los tres tipos de vegetación más importantes para la actividad ganadera en el estado de Chihuahua. En total se estudiaron 29 ranchos ganaderos del sistema vaca-cría, de los cuales, 10 tenían pastizales medianos, 8 pastizales amacollados arborescentes y 11 matorrales (principalmente sub-inermes y sub-espinosos). La condición del pastizal de cada rancho se evaluó mediante el método COTECOCA (1978) y el ICEP, muestreando de 1 a 7 potreros por rancho, dependiendo de la superficie de cada rancho y de la homogeneidad de su vegetación. En cada potrero se muestrearon tres transectos, tanto para la determinación de la cobertura basal de la vegetación como para efectuar las observaciones del ICEP. La cobertura de la vegetación se determinó mediante la técnica de punta de pie en transectos de alrededor de $400 \mathrm{~m}$, a razón de 200 observaciones por transecto. Las determinaciones de la cobertura de vegetación se efectuaron por pasantes de la carrera de Ingeniero Zootecnista en Sistemas de Producción de la Universidad Autónoma de Chihuahua, quienes conformaron dos equipos de trabajo integrados por dos personas. En cada equipo, una persona llevaba a cabo el muestreo de la vegetación y otra registraba los datos recabados. Los sitios de muestreo fueron seleccionados tomando en cuenta su representatividad con respecto a la vegetación dominante en cada rancho bajo estudio. A partir de la composición florística de cada transecto, se identificó el tipo de vegetación correspondiente, con base en la información contenida en las Guías de COTECOCA (1978). Posteriormente, se obtuvieron los porcentajes de las especies presentes, las cuales fueron clasificadas como deseables, menos deseables e indeseables. Finalmente, con base en los criterios descritos en la guía de COTECOCA, se determinó la condición del pastizal en cada uno de los ranchos estudiados. En dicha guía se especifica que una cobertura relativa de $25 \%$ o menos de especies clave (deseables y menos deseables) corresponde a una condición pobre, mayor de $25 \%$ y menor de $50 \%$ a una condición regular, mayor de $50 \%$ y menor de $75 \%$ a una condición buena, y una cobertura mayor de $75 \%$ equivale a una condición excelente.

EI ICEP se elaboró a partir de estimaciones visuales de cuatro indicadores relacionados con la productividad y la estabilidad ecológica de los pastizales. Dichas estimaciones se llevaron a cabo en los mismos transectos en los que se determinó la cobertura vegetal. En cada uno de los transectos, un observador realizaba tres estimaciones, una al principio, una en medio y otra al final del transecto. A cada indicador se le asignó una calificación en la escala de 1 al 4, en la que una calificación de 1 correspondía a una situación pobre o indeseable; 2: regular; 3: buena; 4: excelente. Todas las evaluaciones fueron efectuadas por dos personas, el primero y el segundo autor de este trabajo, con el fin de eliminar posibles sesgos por efecto en la interpretación de los criterios. Los indicadores del ICEP fueron los siguientes: cobertura total de vegetación, cobertura de especies forrajeras perennes, capacidad de retención de agua y estabilidad de suelos. Las calificaciones para cada indicador se otorgaron con base en los criterios descritos en la Tabla 1. El ICEP se determinó mediante el cálculo del promedio de los cuatro indicadores estudiados, de tal modo que a cada indicador se le otorgó el mismo valor de importancia. Para fines de comparación y de análisis estadísticos, los promedios de cada indicador fueron redondeados, para generar valores enteros del 1 al 4. De igual manera, la condición del pastizal se transformó a valores categóricos en la misma escala del 1 al 4, en la que una condición pobre correspondía a un valor de 1 , regular de 2 , buena de 3 , y excelente de 4.

La confiabilidad del ICEP se efectuó comparando sus resultados con los datos correspondientes a la determinación de la condición de los pastizales mediante el método de vegetación clímax o método de COTECOCA, por ser este último el método oficial en México para determinar la condición de los pastizales y los coeficientes de agostadero. Los datos generados por los dos métodos se sometieron a una prueba de independencia de chi cuadrada de Pearson (Franke et al. 2012). Con el objetivo de mostrar gráficamente la relación entre 
Tabla 1. Criterios para otorgar las calificaciones de los indicadores de contribución ecológica de los pastizales.

\begin{tabular}{|c|c|c|c|c|}
\hline & \multicolumn{4}{|c|}{ Calificaciones } \\
\hline & 1 & 2 & 3 & 4 \\
\hline Indicador & \multicolumn{4}{|c|}{ Criterios } \\
\hline $\begin{array}{l}\text { Cobertura gene- } \\
\text { ral de vegetación }\end{array}$ & $\begin{array}{l}\text { Vegetación incipiente, la } \\
\text { gran mayoría de la su- } \\
\text { perficie consta de suelo } \\
\text { desnudo. }\end{array}$ & $\begin{array}{l}\text { Vegetación escasa, dis- } \\
\text { tribuida en forma de } \\
\text { parches, una parte im- } \\
\text { portante de la superficie } \\
\text { consta de suelo desnudo. }\end{array}$ & $\begin{array}{l}\text { Vegetación abundante, } \\
\text { ausencia de parches, la } \\
\text { mayoría del suelo está } \\
\text { cubierta por vegetación. }\end{array}$ & $\begin{array}{l}\text { Vegetación exuberante, } \\
\text { prácticamente todo el } \\
\text { suelo está cubierto por } \\
\text { vegetación. }\end{array}$ \\
\hline $\begin{array}{l}\text { Cobertura de es- } \\
\text { pecies forrajeras } \\
\text { perennes }\end{array}$ & $\begin{array}{l}\text { La gran mayoría de las } \\
\text { plantas son arbustos in- } \\
\text { deseables, los arbustos } \\
\text { forrajeros y los zacates } \\
\text { perennes son escasos o } \\
\text { inexistentes. }\end{array}$ & $\begin{array}{l}\text { La cubierta de } \text { arbus- } \\
\text { tos forrajeros y zacates } \\
\text { perennes es escasa, } \\
\text { predominan } \\
\text { indeseables y } \\
\begin{array}{l}\text { anuales. } \\
\text { andacatos }\end{array} \\
\end{array}$ & 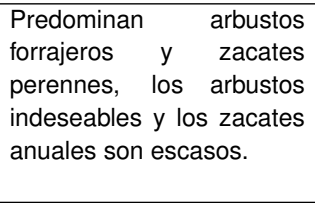 & $\begin{array}{l}\text { La cubierta de especies } \\
\text { forrajeras es abundante, } \\
\text { los arbustos indeseables y } \\
\text { los zacates anuales son } \\
\text { muy escasos o inexis- } \\
\text { tentes. }\end{array}$ \\
\hline $\begin{array}{l}\text { Capacidad de re- } \\
\text { tención de agua }\end{array}$ & $\begin{array}{l}\text { Las evidencias de escu- } \\
\text { rrimientos bajo regímenes } \\
\text { turbulentos como arroyos, } \\
\text { arroyuelos y terracetas son } \\
\text { abundantes. }\end{array}$ & $\begin{array}{l}\text { Las evidencias de escu- } \\
\text { rrimientos bajo regímenes } \\
\text { turbulentos como canales, } \\
\text { canalillos y terracetas son } \\
\text { frecuentes. }\end{array}$ & $\begin{array}{l}\text { Las evidencias de escu- } \\
\text { rrimientos bajo regímenes } \\
\text { turbulentos como arroyos, } \\
\text { arroyuelos y terracetas son } \\
\text { escasas. }\end{array}$ & $\begin{array}{l}\text { No se encuentran eviden- } \\
\text { cias de escurrimientos tur- } \\
\text { bulentos }\end{array}$ \\
\hline $\begin{array}{l}\text { Estabilidad } \\
\text { suelos }\end{array}$ & $\begin{array}{l}\text { Las evidencias de erosión } \\
\text { del suelo como canales, } \\
\text { canalillos, pedestales y } \\
\text { cárcavas son abundantes. }\end{array}$ & $\begin{array}{l}\text { Las evidencias de erosión } \\
\text { del suelo como canales, } \\
\text { canalillos, pedestales y } \\
\text { cárcavas son frecuentes. }\end{array}$ & $\begin{array}{l}\text { Las evidencias de erosión } \\
\text { del suelo como canales, } \\
\text { canalillos, pedestales y } \\
\text { cárcavas son escasas. }\end{array}$ & $\begin{array}{l}\text { No se encuentran eviden- } \\
\text { cias de erosión del suelo. }\end{array}$ \\
\hline
\end{tabular}

los dos métodos de evaluación, se llevó a cabo un análisis de correspondencias (Ter Braak 1986). Para modelar la relación entre los valores del ICEP y los de condición del pastizal, se efectuó un análisis de regresión logística ordinal (McCullagh 1980, Agresti 1990), utilizando el estadístico gama de GoodmanKruskal para cuantificar la magnitud de la asociación entre los valores del ICEP y los de condición del pastizal (Goodman y Kruskal 1954). Todos los análisis estadísticos fueron efectuados separando los datos por tipo de vegetación, excepto el de regresión logística ordinal, en el que se usaron los datos de los 29 ranchos.

El grado de precisión en la estimación del ICEP se evaluó mediante un ensayo de valoración del grado de objetividad de los criterios que constituyeron la base para el otorgamiento de las calificaciones. El ensayo consistió en la evaluación de un rancho, la cual se llevó a cabo con la participación de nueve observadores, quienes otorgaron sus calificaciones a cada uno de los cuatro indicadores. La evaluación se llevó a cabo en el Rancho El Jeromín, el cual está ubicado en el municipio de Aldama, Chihuahua. Los nueve observadores eran investigadores del Instituto Nacional de Investigaciones Forestales, Agrícolas y Pecuarias, quienes contaban con una experiencia de más de 25 años en trabajos de investigación relacionados con el manejo de ganado bovino y de recursos naturales, particularmente en lo relativo a vegetación de pastizales. Con el fin de evitar alguna influencia sobre los observadores, el proceso de calificación se efectuó sin llevar a cabo un proceso de entrenamiento previo que permitiera homogeneizar los criterios de calificación, a cada observador sólo se le ofreció una breve explicación sobre el objetivo de la evaluación y sobre cómo se llevaría a cabo. Una vez efectuada la evaluación, las calificaciones se tabularon conformando una matriz de indicadores y observadores. La confiabilidad de la calificación otorgada a cada uno de los indicadores del ICEP se efectuó determinando las diferencias entre evaluadores, partiendo de la hipótesis de que la diferencia entre las calificaciones se debe exclusivamente a la interpretación individual de los criterios, dado que todos los observadores evaluaron el mismo rancho. Para evaluar la facilidad de aplicación de los criterios de asignación de calificaciones para cada uno de los cuatro indicadores del ICEP se llevó a cabo un ensayo de evaluación en el Rancho Charco Azul, municipio de Parral, Chihuahua. En dicho ensayo participaron 11 personas con diferente formación académica y ocupación. De los 11 obser- 
vadores, cinco eran técnicos del área agropecuaria, dos profesionistas del área agropecuaria, dos secretarias de oficina, un productor y el primer autor de este trabajo.

\section{RESULTADOS}

No se obtuvieron evidencias estadísticas que sustenten la independencia entre los datos generados mediante el método propuesto y los del método tradicional ( $p>0.05$; Chi cuadrada de Pearson) en los tres tipos de vegetación estudiados. De igual manera, mediante los análisis de correspondencias y de regresión logística ordinal (gama de GoodmanKruskal $=0.91, p<0.01)$ se demostró la existencia de una relación estrecha entre los valores del ICEP y los valores correspondientes a la condición del pastizal (Figura 1 y Tabla 2). En consecuencia, los dos métodos generan los mismos resultados, en términos de precisión y confiabilidad. Sin embargo, el método propuesto ofrece una información más completa, toda vez que, además del parámetro de la vegetación, el ICEP integra los datos relativos a la estabilidad de los suelos y la capacidad de retención de agua de los pastizales. Adicionalmente, el ICEP ofrece la ventaja de ser un método más eficiente en el levantamiento de la información, ya que esa labor es efectuada por una sola persona, en tanto que en el levantamiento de los datos de cobertura vegetal se requiere de la participación de dos personas y el tiempo de muestreo en ambas opciones es aproximadamente el mismo.

En términos generales, las calificaciones asignadas al ICEP en cada potrero de los pastizales medianos resultaron muy parecidas en relación con el valor correspondiente a la condición (Tabla 3). La media general del valor otorgado a la condición fue de 2.4, en tanto que el valor del ICEP fue de 2.3, con errores estándar de \pm 0.10 y \pm 0.09 , respectivamente. Como era de esperarse, las medias de los valores redondeados del índice y de la condición también fueron similares, al igual que sus errores estándar. En la misma tabla también se observa que la media general de la cobertura relativa de especies clave en los pastizales medianos fue de $47.11 \%$, la cual corresponde a una condición regular, cercana a la condición buena, lo que es concomitante con los valores categóricos de condición y del ICEP.

La Tabla 4 contiene los resultados obtenidos en los ranchos de pastizales amacollados. Se puede apreciar que existe una alta similaridad entre los valores otorgados a la condición y al ICEP. De igual manera, se puede observar una alta asociación entre la cobertura relativa de especies clave y la condición que a esa cobertura le corresponde, así como con respecto a las medias de los valores antes mencionados. De acuerdo con su cobertura relativa de vegetación, cuya media general fue de $53.37 \%$, a los ranchos de los pastizales amacollados les corresponde una condición buena. No obstante, todos los valores absolutos y redondeados de la condición y del ICEP se situaron por debajo de 3, que es el valor que le corresponde a un pastizal en condición buena.

Los resultados de cobertura relativa, de condición y del ICEP de los ranchos con vegetación de matorrales aparecen en la Tabla 5. Es notorio que la condición en los ranchos con matorrales está más deteriorada en comparación con los de pastizales medianos y los amacollados. La media de su cobertura relativa de especies clave fue de $31.60 \%$ y le corresponde una clasificación de condición regular. Sobresale la información del rancho Los Arenales, en el que algunos de sus potreros tuvieron una alta cobertura de vegetación y, por ende, mejor condición. Es importante señalar que en los potreros de mejor condición en el Rancho Los Arenales dominaba el zacate toboso (Hilaria mutica), el cual es conocido por su alto contenido fibroso y su resistencia al pastoreo. Los valores absolutos y redondeados de la condición y del ICEP se situaron por debajo de los valores que le corresponden con respecto al estado en que se encontraban los ranchos de matorrales. Eso también se reflejó en las medias de los valores redondeados de condición e ICEP que fueron de 1.9 y 1.8, respectivamente, que corresponden a una condición pobre, a pesar de que la condición en general era regular, próxima a pobre.

La Tabla 6 contiene las calificaciones otorgadas a los cuatro indicadores del ICEP por nueve observadores (investigadores). En dicha tabla se 


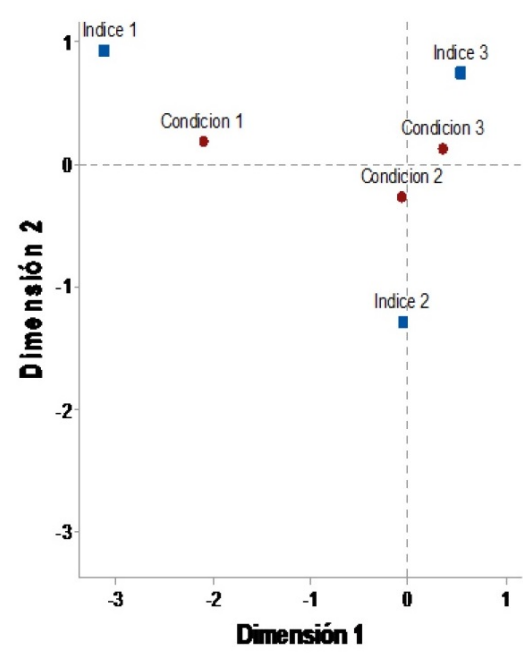

Pastizales medianos

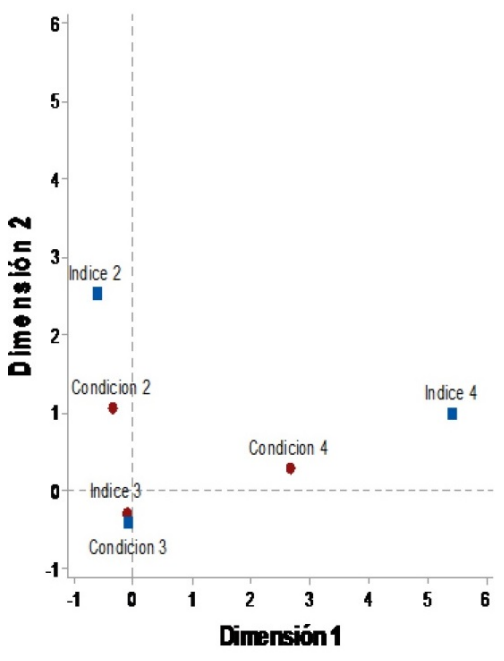

Pastizales amacollados

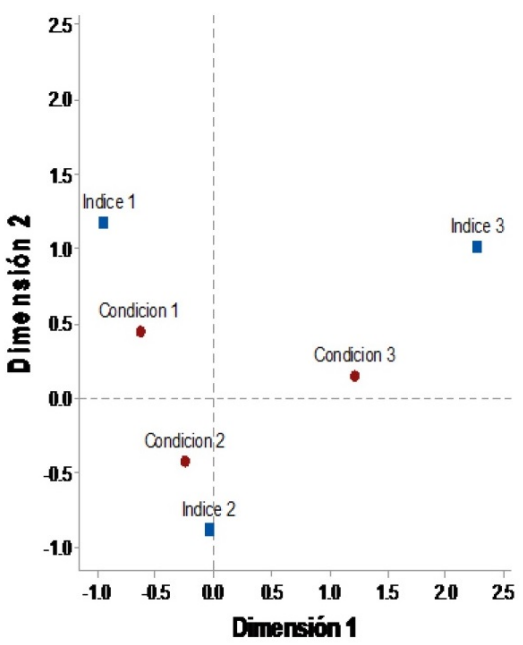

Matorrales

Figura 1. Análisis de correspondencias sobre la relación entre los valores del ICEP y los valores otorgados a la condición del pastizal.

Tabla 2. Parámetros de la prueba de regresión logística ordinal sobre la relación entre los datos del ICEP y los de condición del pastizal.

\begin{tabular}{lccccc}
\hline Parámetro & Grados de libertad & Estimador & Error estándar & Chi-cuadrada & P-value \\
\hline Intercepto 1 & 1 & 3.4495 & 0.717 & 23.146 & $<0.0001$ \\
Intercepto 2 & 1 & 6.4338 & 0.9205 & 48.8538 & $<0.0001$ \\
Intercepto 3 & 1 & 11.5581 & 1.3431 & 74.0593 & $<0.0001$ \\
Índice & 1 & -2.7198 & 0.3752 & 52.5522 & $<0.0001$ \\
\multicolumn{5}{c}{ Pruebas de bondad del ajuste } \\
Método & Chi cuadrada & GL & P-value & \\
Pearson & 3.94968 & 8 & 0.86 & \\
Desviación & 5.07211 & 8 & 0.75 & \\
Grados de libertad & Estadístico de prueba & P-value & & \\
1 & 76.887 & $<0.0001$ & \\
Gamma de Goodman-Kruskal= 0.91 & Medidas de asociación \\
Criterio de información de Akaike $=167.3$ &
\end{tabular}

puede apreciar que la mayoría de las calificaciones para los indicadores de cobertura general de vegetación y cobertura de especies forrajeras fluctuaron entre 1 y 2 . Con estos valores se puede deducir que el terreno se encuentra en condición de pobre a regular. Las calificaciones en los indicadores de estabilidad de suelos y de capacidad de retención de agua fluctuaron entre 1 y 3 , predominando 1 y 2 . Estos valores revelan que ambos atributos se encuentran también en una condición de regular a pobre. Los errores estándar presentan valores bajos, lo que es indicativo de un aceptable grado de confiabilidad de los criterios de calificación. Los datos muestran también que resultó más homogénea la calificación de los atributos de vegetación (cobertura general y cobertura de especies clave) que la calificación otorgada a los atributos derivadas de retención de agua y estabilidad de suelos.

En La Tabla 7 se presentan los resultados de la evaluación efectuada por observadores de diferente formación académica y ocupación. Se puede apreciar que existe una alta homogeneidad en la interpretación de los criterios de asignación de calificaciones, resultando un índice general de 3.9 y un error 
Tabla 3. Cobertura relativa de especies clave, condición e índice de contribución ecológica en los ranchos de pastizales medianos.

\begin{tabular}{|c|c|c|c|c|c|c|}
\hline Rancho & Potrero & Cobertura $^{1}$ & Condición ${ }^{2}$ & Indice ${ }^{3}$ & Condición ${ }^{4}$ & Indice 5 \\
\hline \multirow{3}{*}{ Tres Hermanos } & 1 & 41.75 & 2.2 & 3.1 & 2 & 3 \\
\hline & 2 & 24.25 & 1.5 & 1.6 & 2 & 2 \\
\hline & 3 & 29.00 & 2 & 2.8 & 2 & 3 \\
\hline \multirow{4}{*}{ Amparanes } & 1 & 44.50 & 2.5 & 2.9 & 3 & 3 \\
\hline & 2 & 44.25 & 2 & 2.8 & 2 & 3 \\
\hline & 3 & 36.25 & 2 & 2.4 & 2 & 2 \\
\hline & 4 & 52.00 & 2.5 & 2.8 & 3 & 3 \\
\hline \multirow{3}{*}{ Barranco Blanco } & 1 & 34.25 & 1.7 & 1.5 & 2 & 2 \\
\hline & 2 & 30.38 & 2 & 1.9 & 2 & 2 \\
\hline & 1 & 50.75 & 2.5 & 2.6 & 3 & 3 \\
\hline \multirow{3}{*}{ Charco Largo } & 2 & 58.50 & 2.5 & 2.7 & 3 & 3 \\
\hline & 3 & 47.50 & 2.5 & 2.7 & 3 & 3 \\
\hline & 4 & 51.00 & 2.5 & 2.6 & 3 & 3 \\
\hline \multirow[t]{2}{*}{ El Arbolito } & Único & 54.00 & 2.5 & 2.8 & 3 & 3 \\
\hline & 1 & 45.50 & 2 & 2.8 & 2 & 3 \\
\hline \multirow[t]{3}{*}{ El Chaparral } & 2 & 57.50 & 3 & 2.9 & 3 & 3 \\
\hline & 3 & 50.25 & 2.5 & 3.0 & 3 & 3 \\
\hline & 1 & 43.00 & 2 & 3.0 & 2 & 3 \\
\hline \multirow{3}{*}{ Franqueño } & 2 & 24.50 & 1 & 1.3 & 1 & 1 \\
\hline & 3 & 16.50 & 1 & 1.5 & 1 & 2 \\
\hline & 4 & 19.50 & 1 & 1.5 & 1 & 1 \\
\hline \multirow[t]{3}{*}{ San Rafael } & Único & 61.83 & 3 & 3.0 & 3 & 3 \\
\hline & 1 & 41.83 & 2 & 1.4 & 2 & 1 \\
\hline & 2 & 40.08 & 2 & 1.7 & 2 & 2 \\
\hline \multirow{4}{*}{ Agua nueva } & 3 & 66.00 & 3 & 2.0 & 3 & 2 \\
\hline & 4 & 63.92 & 3 & 2.1 & 3 & 2 \\
\hline & 5 & 40.08 & 1.8 & 1.4 & 2 & 1 \\
\hline & 6 & 48.17 & 2.3 & 1.8 & 2 & 2 \\
\hline \multirow{8}{*}{ Loren } & 1 & 57.33 & 3 & 1.8 & 3 & 2 \\
\hline & 2 & 50.75 & 2.5 & 2.7 & 3 & 3 \\
\hline & 3 & 51.00 & 3 & 2.0 & 3 & 2 \\
\hline & 4 & 63.67 & 3 & 2.0 & 3 & 2 \\
\hline & 5 & 68.50 & 3.3 & 2.5 & 3 & 3 \\
\hline & 6 & 58.17 & 3 & 2.3 & 3 & 2 \\
\hline & 7 & 65.00 & 3.3 & 2.8 & 3 & 3 \\
\hline & 8 & 64.67 & 3 & 2.8 & 3 & 3 \\
\hline Media & - & 47.11 & 2.4 & 2.3 & 2.5 & 2.4 \\
\hline Error estándar & - & 2.26 & 0.10 & 0.09 & 0.11 & 0.11 \\
\hline
\end{tabular}

estándar de 0.05. Comparando los datos de los dos grupos de evaluadores, resulta evidente que es más fácil evaluar un terreno en condición buena a excelente, en comparación a uno que se encuentra en condición pobre o regular.

\section{DISCUSIÓN}

El uso de índices para la estimación de la condición de los pastizales ha sido de gran interés a través del tiempo, por ejemplo, Ludwig et al. (2007) propusieron un índice de pérdida de recursos el cual fue estimado con la cobertura vegetal medida a través de sensores remotos y la altitud de los sitios de pastizales en Australia. Concluyendo que este índice es útil para la detección de cambios en la condición de los pastizales abiertos y que no es recomendable para sitios con cobertura aérea de árboles y/o arbustos. Al respecto, en nuestro trabajo, la cobertura vegetal total y de especies perennes son parte crucial para la integración del ICEP, lo que coincide con Sanaei et al. (2018) quienes indican que la 
Tabla 4. Cobertura relativa de especies clave, condición e índice de contribución ecológica en los ranchos de pastizales amacollados.

\begin{tabular}{|c|c|c|c|c|c|c|}
\hline Rancho & Potrero & Cobertura $^{1}$ & Condición ${ }^{2}$ & Indice ${ }^{3}$ & Condición ${ }^{4}$ & Indice ${ }^{5}$ \\
\hline \multirow{4}{*}{ Charco azul } & 1 & 57.25 & 3 & 2.5 & 3 & 3 \\
\hline & 2 & 62.5 & 3 & 3.0 & 3 & 3 \\
\hline & 3 & 56.75 & 2.5 & 2.6 & 3 & 3 \\
\hline & 4 & 53.25 & 2.5 & 2.7 & 3 & 3 \\
\hline \multirow{4}{*}{ Cima de San A. } & 1 & 56.75 & 3 & 2.7 & 3 & 3 \\
\hline & 2 & 53.00 & 2.5 & 2.8 & 3 & 3 \\
\hline & 3 & 52.00 & 2.5 & 2.5 & 3 & 3 \\
\hline & 4 & 60.25 & 3 & 2.8 & 3 & 3 \\
\hline \multirow{4}{*}{ El Mimbre } & 1 & 36.75 & 2 & 2.9 & 2 & 3 \\
\hline & 2 & 47.00 & 2.5 & 3.0 & 3 & 3 \\
\hline & 3 & 55.75 & 2.5 & 3.0 & 3 & 3 \\
\hline & 4 & 51.00 & 2.5 & 3.0 & 3 & 3 \\
\hline \multirow{4}{*}{ San Andrés } & 1 & 53.25 & 3 & 3.3 & 3 & 3 \\
\hline & 2 & 52.75 & 2.5 & 2.8 & 3 & 3 \\
\hline & 3 & 59.75 & 3 & 3.2 & 3 & 3 \\
\hline & 4 & 52.00 & 2.5 & 2.8 & 3 & 3 \\
\hline \multirow{3}{*}{ San Antonio } & 1 & 24.00 & 1.5 & 2.1 & 2 & 2 \\
\hline & 2 & 61.00 & 3 & 2.5 & 3 & 3 \\
\hline & 3 & 52.75 & 2.5 & 2.0 & 3 & 2 \\
\hline \multirow{3}{*}{ San Ignacio } & 1 & 44.25 & 2 & 2.7 & 2 & 3 \\
\hline & 2 & 32.00 & 2 & 1.8 & 2 & 2 \\
\hline & 3 & 54.50 & 2.5 & 3.0 & 3 & 3 \\
\hline \multirow{3}{*}{ San Ignacio C. } & 1 & 65.25 & 3 & 2.8 & 3 & 3 \\
\hline & 2 & 49.00 & 2 & 2.5 & 2 & 3 \\
\hline & 3 & 41.00 & 2 & 2.5 & 2 & 2 \\
\hline \multirow{6}{*}{ Bajío de Tónachi } & 1 & 56.50 & 3 & 2.9 & 3 & 3 \\
\hline & 2 & 83.75 & 3.5 & 3.3 & 4 & 3 \\
\hline & 3 & 45.75 & 2.5 & 3.0 & 3 & 3 \\
\hline & 4 & 75.00 & 3.5 & 3.6 & 4 & 4 \\
\hline & 5 & 48.50 & 2.5 & 3.2 & 3 & 3 \\
\hline & 6 & 61.25 & 3 & 2.7 & 3 & 3 \\
\hline Media & & 53.37 & 2.6 & 2.8 & 2.9 & 2.9 \\
\hline Error estándar & & 2.03 & 0.08 & 0.07 & 0.09 & 0.07 \\
\hline
\end{tabular}

cobertura vegetal tiene un alto impacto en la diversidad de especies y la biomasa aérea del pastizal y puede ser usado como indicador ecológico para el monitoreo de la conservación de la biodiversidad, así como el funcionamiento de los ecosistemas de pastizales. Mientras que Sinclair et al. (2021) proponen la estimación de la condición de los pastizales a través del consenso de un grupo de gente interesada en los pastizales incluyendo pastores nómadas, botánicos, ecólogos y tomadores de decisiones.

Las diferencias entre la determinación de la condición a partir de la cobertura relativa de vegetación y la obtenida a través de la asignación de un índice pueden ser atribuidas al hecho de que el método de vegetación clímax se basa en una es- cala de valores de 0 a 100, con rangos de 25 puntos. La utilización de una escala tan amplia implica que a un pastizal con un $49 \%$ de cobertura le corresponde una condición regular; es decir, por un punto porcentual no recibe la categoría de condición buena. En contraparte, en la metodología propuesta se utiliza una escala numérica menor y, en lugar de expresar la condición del pastizal en términos cualitativos, ésta se define por un coeficiente, lo que lo hace más preciso y confiable (Ahmadpour et al. 2016). Las diferencias con relación al método tradicional (Andrade et al. 2019) también refrendan la robustez y la importancia del ICEP. En el caso de los ecosistemas de matorrales, un sitio en particular puede encontrase en condición regular dada la presencia de una 
Tabla 5. Cobertura relativa de especies clave, condición e índice de contribución ecológica en los ranchos de matorrales

\begin{tabular}{|c|c|c|c|c|c|c|}
\hline Rancho & Potrero & Cobertura $^{1}$ & Condición ${ }^{2}$ & Indice $^{3}$ & Condición ${ }^{4}$ & Indice 5 \\
\hline \multirow{4}{*}{ Agua Prieta } & 1 & 11.00 & 1.0 & 1.3 & 1 & 1 \\
\hline & 2 & 19.50 & 1.0 & 1.7 & 1 & 2 \\
\hline & 3 & 19.00 & 1.0 & 1.8 & 1 & 2 \\
\hline & 4 & 22.75 & 1.5 & 1.5 & 2 & 2 \\
\hline \multirow{4}{*}{ Agua Prieta II } & 1 & 12.75 & 1.0 & 1.5 & 1 & 1 \\
\hline & 2 & 36.25 & 2.0 & 1.3 & 2 & 1 \\
\hline & 3 & 13.75 & 1.0 & 1.2 & 1 & 1 \\
\hline & 4 & 40.25 & 2.5 & 2.4 & 3 & 2 \\
\hline \multirow{4}{*}{ El Edén } & 1 & 28.25 & 1.5 & 2.0 & 2 & 2 \\
\hline & 2 & 54.25 & 2.5 & 2.9 & 3 & 3 \\
\hline & 3 & 45.25 & 2.3 & 2.4 & 2 & 2 \\
\hline & 4 & 25.50 & 1.5 & 2.1 & 2 & 2 \\
\hline \multirow{2}{*}{ El Palo Chino } & 1 & 14.25 & 1.0 & 1.4 & 1 & 1 \\
\hline & 2 & 18.25 & 1.5 & 1.5 & 2 & 1 \\
\hline \multirow{3}{*}{ La Cieneguilla } & 1 & 18.25 & 1.0 & 1.5 & 1 & 1 \\
\hline & 2 & 11.00 & 1.0 & 1.1 & 1 & 1 \\
\hline & 3 & 23.25 & 1.5 & 2.2 & 2 & 2 \\
\hline \multirow{4}{*}{ La Estacada } & 1 & 47.5 & 2.5 & 2.3 & 3 & 2 \\
\hline & 2 & 43.25 & 2.0 & 2.3 & 2 & 2 \\
\hline & 3 & 53.75 & 3.0 & 2.4 & 3 & 2 \\
\hline & 4 & 47.50 & 2.5 & 2.4 & 362 & \\
\hline \multirow{4}{*}{ La Hebilla } & 1 & 36.25 & 2.0 & 1.4 & 2 & 1 \\
\hline & 2 & 28.25 & 1.5 & 1.6 & 2 & 2 \\
\hline & 3 & 25.75 & 1.5 & 1.5 & 2 & 1 \\
\hline & 4 & 24.75 & 1.5 & 2.2 & 2 & 2 \\
\hline \multirow{4}{*}{ Los Ojuelos } & 1 & 15.25 & 1.0 & 2.0 & 1 & 2 \\
\hline & 2 & 7.00 & 1.0 & 1.0 & 1 & 1 \\
\hline & 3 & 40.75 & 2.5 & 2.7 & 3 & 3 \\
\hline & 4 & 12.50 & 1.0 & 1.5 & 1 & 2 \\
\hline \multirow{4}{*}{ Los Walters } & 1 & 13.25 & 1.0 & 1.4 & 1 & 1 \\
\hline & 2 & 20.25 & 1.0 & 1.5 & 1 & 2 \\
\hline & 3 & 23.5 & 1.0 & 1.5 & 1 & 1 \\
\hline & 4 & 22.00 & 1.5 & 1.8 & 2 & 2 \\
\hline \multirow{3}{*}{ San Lorenzo } & 1 & 37.25 & 2.0 & 1.8 & 2 & 2 \\
\hline & 2 & 26.50 & 1.5 & 2.1 & 2 & 2 \\
\hline & 3 & 20.25 & 1.5 & 2.0 & 2 & 2 \\
\hline \multirow{7}{*}{ Los arenales } & 1 & 63.83 & 3.0 & 2.8 & 3 & 3 \\
\hline & 2 & 64.67 & 3.0 & 2.5 & 3 & 3 \\
\hline & 3 & 74.00 & 3.3 & 2.8 & 3 & 3 \\
\hline & 4 & 61.00 & 2.8 & 2.3 & 3 & 2 \\
\hline & 5 & 25.33 & 1.5 & 2.0 & 2 & 2 \\
\hline & 6 & 60.50 & 2.8 & 2.7 & 3 & 3 \\
\hline & 7 & 50.67 & 2.3 & 1.8 & 2 & 2 \\
\hline Media & - & 31.60 & 1.7 & 1.9 & 1.9 & 1.8 \\
\hline Error estándar & - & 2.67 & 0.11 & 0.08 & 0.12 & 0.09 \\
\hline
\end{tabular}

buena población de arbustos forrajeros. Esta clasificación se daría a pesar de que la cubierta de zacates perennes fuese mínima, de tal manera que se presentan mayores evidencias de escurrimientos turbulentos del agua de lluvia y mayores grados de erosión de los suelos (Chartier y Rostagno 2006) por lo que su condición, en términos del ICEP, posiblemente resultaría pobre y no regular. Por el contrario, sitios con una buena cubierta de especies no deseables 0 menos deseables pueden ser clasificados en condición pobre con base en la vegetación clímax, a pesar del papel que esas especies desempeñan en térmi- 
Tabla 6. Calificaciones otorgadas por nueve observadores (investigadores) en la estimación de los indicadores del ICEP. Rancho El Jeromín.

\begin{tabular}{lccccccccccc}
\hline & \multicolumn{10}{c}{ Observador } \\
\cline { 2 - 10 } Indicador & 1 & 2 & 3 & 4 & 5 & 6 & 7 & 8 & 9 & Media & EE $^{1}$ \\
\hline Cobertura de vegetación & $1^{2}$ & 2 & 2 & 2 & 2 & 2 & 2 & 2 & 2 & 1.89 & 0.1 \\
Cobertura de especies clave & 1 & 1 & 2 & 2 & 2 & 1 & 2 & 2 & 2 & 1.67 & 0.2 \\
Retención de agua & 2 & 1 & 2 & 3 & 3 & 1 & 2 & 2 & 3 & 2.11 & 0.3 \\
Estabilidad de suelos & 2 & 1 & 3 & 3 & 2 & 2 & 2 & 3 & 3 & 2.33 & 0.2 \\
\hline Indice $^{3}$ & 1.5 & 1.2 & 2.2 & 2.0 & 2.2 & 1.5 & 2.0 & 2.2 & 2.0 & $2.00^{4}$ & 0.2 \\
\hline${ }^{1}$ Error estándar, ${ }^{2}$ Calificaciones otorgadas por los diferentes observadores, ${ }^{3}$ Media de los cuatro indicadores \\
dentro de observadores, ${ }^{4}$ Media de medias.
\end{tabular}

Tabla 7. Calificaciones otorgadas por once observadores (diferentes ocupaciones y grado académico) en la estimación de los indicadores del ICEP. Rancho Charco Azul.

\begin{tabular}{lccccccccccccc}
\hline & \multicolumn{11}{c}{ Observador } & & \\
\cline { 2 - 12 } Indicador & 1 & 2 & 3 & 4 & 5 & 6 & 7 & 8 & 9 & 10 & 11 & Media & EE $^{1}$ \\
\hline Cobertura de vegetación & $3^{2}$ & 4 & 4 & 4 & 3 & 3 & 4 & 4 & 3 & 4 & 4 & 3.6 & 0.15 \\
Cobertura de especies clave & 4 & 4 & 4 & 4 & 3 & 4 & 4 & 4 & 4 & 4 & 4 & 3.9 & 0.09 \\
Retención de agua & 4 & 4 & 4 & 4 & 4 & 4 & 4 & 4 & 4 & 4 & 4 & 4 & 0 \\
Estabilidad de suelos & 4 & 4 & 4 & 4 & 4 & 4 & 4 & 4 & 4 & 4 & 4 & 4 & 0 \\
\hline Índice $^{3}$ & 3.7 & 4 & 4 & 4 & 3.5 & 3.7 & 4 & 4 & 3.7 & 4 & 4 & $3.9^{4}$ & 0.05 \\
\hline
\end{tabular}

${ }^{1}$ Error estándar, ${ }^{2}$ Calificaciones otorgadas por los diferentes observadores, ${ }^{3}$ Media de los cuatro indicadores dentro de observadores, ${ }^{4}$ Media de medias.

nos de la preservación de hábitat para diversas especies animales y en la captura de carbono (McKeon et al. 2009, McSherry y Ritchie 2013). De acuerdo con Perkins et al. (2013) el uso del concepto de condición del pastizal es inadecuado por su carácter reduccionista, por lo que debería ser sustituido por una medición basada en las características del suelo, como medio para determinar el éxito del manejo en la conservación del sitio, no sólo desde el punto de vista productivo sino también ecológico y ambiental. Se debe recordar que en los suelos se encuentra almacenado el doble del carbono (Ricard y Viglizzo 2020) en comparación al que se encuentra en la atmósfera, de tal manera que un uso adecuado de los pastizales a través del pastoreo contribuye al sostenimiento de un buen estado de la vegetación, del suelo, de la retención del agua y, por lo tanto, de una mayor capacidad de fijación de carbono (McSherry y Ritchie 2013, Abdalla et al. 2018).

Una ventaja importante del ICEP es que el pastizal se evalúa sin la necesidad de contrastar lo observado contra un estado ideal o potencial, tal y como se hace en el método tradicional. En el caso del método del estado de salud (Pyke et al. 2002), los autores refieren que las diferencias conceptuales y, por lo tanto, la puesta en práctica del es- tado ideal teórico conduce a una importante variación en los criterios de evaluación, por lo que la homologación entre observadores con respecto a dichos puede tomar hasta tres años de práctica y entrenamiento. En el caso del ICEP, la evaluación se efectúa con base en la percepción de la persona evaluadora, cuya certeza depende de la aplicación de los criterios de evaluación. Aunque en este estudio no se encontraron diferencias en la interpretación y la aplicación de los criterios de evaluación por efecto de la formación académica del evaluador, resulta evidente que a mayor experiencia y formación académica idónea le corresponden mejores niveles de calidad en la evaluación. Al respecto, Abate (2016) especificó que los ganaderos, a lo largo de su vida y con la observación cotidiana de sus ranchos ganado, han desarrollado la capacidad de detectar cambios y describir el estado productivo de sus pastizales, lo que los sitúa como potenciales buenos usuarios del ICEP. De manera similar, Sayre et al. (2012) hipotetizaron que en el progreso del estado del arte en la ciencia de pastizales y, por lo tanto, en su enriquecimiento, ha sido muy importante la incorporación de conocimientos locales, provenientes de experiencias personales o derivados de experimentos empíricos. Los ganaderos tienen la capaci- 
dad para determinar las causas de los cambios ambientales y relacionarlas con los suelos y la cubierta vegetal, así como describir diferentes tipos de gradientes de degradación, señalando las áreas que están en mayor riesgo de degradación. Todos estos conocimientos son cruciales para construir una línea base de información sobre los pastizales, de tal modo que los ecólogos, los tomadores de decisiones y los técnicos las aprovechen para diseñar de mejor manera las políticas públicas y el desarrollo de planes de cooperación con los productores (Oba 2012). Otros autores también han comprobado que el conocimiento ecológico local de los ganaderos es importante para integrarse como otro indicador en la estimación de la condición y/o monitoreo de los pastizales (Kong et al. 2015, Eddy et al. 2017).

Un método de evaluación de la condición de pastizales propuesto por Sinclair et al. (2021) tiene la desventaja de que maneja un gran número de indicadores (14) y todos ellos se basan solamente en características de la vegetación, sin tomar en cuenta la estabilidad del suelo $u$ otros indicadores ecológicos. Además, estos investigadores encontraron un alto grado de redundancia entre los indicadores, por lo que concluyen que se puede evaluar la condición del pastizal con menos indicadores. Por el contrario, el ICEP solamente toma en cuenta 4 indicadores, lo cual lo convierte en un método práctico y fácil de aplicar. Una limitante del ICEP es que requiere de mediciones en el sitio de estudio, por lo que produce información válida solamente para ese sitio. Los ganaderos, ecologistas e investigadores requieren de información a mayor escala. Ante tal situación, una opción podría ser combinar el ICEP con la información generada mediante sensores remotos (como datos satelitales), para generar información a mayor escala. Si bien las variables con las que se genera el ICEP no pueden ser estimadas a través del uso de sensores remotos, se pueden utilizar sitios evaluados mediante el ICEP para calibrar modelos que puedan predecir la salud de los pastizales a mayor escala (Sinclair et al. 2015, Jones et al. 2018).

\section{CONCLUSIONES}

El índice de contribución ecológica de los pastizales constituye un método confiable, preciso y rápido para llevar a cabo labores de evaluación y monitoreo del estado de salud de los pastizales. Se recomienda que para su implementación se lleve a cabo un proceso de capacitación y entrenamiento, lo que facilitará su aplicación por cualquier persona, independientemente de su formación académica y ocupación.

\section{LITERATURA CITADA}

Abate T (2016) Indigenous ecological knowledge and pastoralist perception on rangeland management and degradation in Guji zone of South Ethiopia. Consilience: The Journal of Sustainable Development 15: 192-218.

Abdalla M, Hastings A, Chadwick DR, Jones DL, Evans DE, Rees RM, Smith P (2018) Critical review of the impacts of grazing intensity on soil organic carbon storage and other soil quality indicators in extensively managed grasslands. Agriculture, Ecosystems and Environment 253: 62-81.

Agresti A (1990) Categorical Data Analysis. John Wiley \& Sons. New York, USA. 394p.

Ahmadpour A, Heshmati GA, Joulaie R (2016) Rangeland condition assessment based on economic criteria. Journal of Landscape Ecology 9: 83-96.

Andrade BO, Boldrini II, Cadenazzi M, Pilar VD, Overbeck GE (2019) Grassland vegetation sampling-a practical guide for sampling and data analysis. Acta Botanica Brasilica 33: 786-795.

Chartier MP, Rostagno CM (2006) Soil erosion thresholds and alternative states in Northeastern Patagonian rangelands. Rangeland Ecology \& Management 59: 616-624. 
COTECOCA (1978) Comisión técnico consultiva para la determinación regional de los coeficientes de agostadero de Chihuahua. SARH. México. 68p.

Dyksterhuis EJ (1949) Condition and management of rangelands based on quantitative ecology. Journal of Range Management 2: 104-115.

Eddy I, Gergel SE, Coops N, Henebry GM, Levine J, Zerriff H, Shibkov E (2017) Integrating remote sensing and local ecological knowledge to monitor rangeland dynamics. Ecological Indicators 82: 106-116.

Franke TM, Ho T, Christie CA (2012) The chi-square test: often used and more often misinterpreted. American Journal of Evaluation 33: 448-458.

Fuhlendorf SD, Engle DM (2001) Restoring heterogeneity on rangelands: ecosystem management based on evolutionary grazing patterns. BioScience 51: 625-632.

Goodman LA, Kruskal WH (1954) Measures of association for cross classifications. Journal of the American Statistic Association 49: 732-764.

Han D, Wang G, Xue B, Liu T, Yinglan A, Xu X (2018) Evaluation of semiarid grassland degradation in North China from multiple perspectives. Ecological Engineering 112: 41-50.

Jones MO, Allred BW, Naugle DE, Maestas JD, Donnelly P, Metz LJ, Karl J, Smith R, Bestelmeyer B, Boyd C, Kerby J, Mclver JD (2018) Innovation in rangeland monitoring: annual, $30 \mathrm{~m}$, plant functional type percent cover maps for US rangelands, 1984-2017. Ecosphere 9: Article e02430. DOI: 10.1002/ecs2.2430.

Karlin M, Galan R, Contreras AMdelC, Zapata R, Coirin R, Ruiz PE (2013) Exergetic model of secondary successions for plant communities in arid Chaco (Argentina). ISRN Biodiversity 2013: Article ID 945190. DOI: $10.1155 / 2013 / 945190$.

Kong TM, Marsh SE, Van Rooven AF, Kellner K, Orr BJ (2015) Assessing rangeland condition in the Kalahari Duneveld through local ecological knowledge of livestock farmers and remotely sensed data. Journal of Arid Environments 113: 77-86.

Ludwig JA, Bastin GN, Chewings VH, Eager RW, Liedloff AC (2007) Leakiness: a new index for monitoring the health of arid and semiarid landscapes using remotely sensed vegetation cover and elevation data. Ecological Indicators 7: 442-454.

Ludwig JA, Bastin GR (2008) Rangeland condition: its meaning and use. A Discussion paper prepared for the Australian Collaborative Rangelands Information System (ACRIS) Management Committee. Australia. 88p.

Maes J, Liquete C, Teller A, Erhard M, Paracchini ML, Barredo JI, Grizzetti B, Cardoso A, Somma F, Petersen JE (2016) An indicator framework for assessing ecosystem services in support of the EU biodiversity strategy 2020. Ecosystem Services 17: 14-23.

McKeon GM, Stone GS, Syktus JI, Carter JO, Flood NR, Ahrens DG, Bruget DN, Chilcott CR, Cobon DH, Cowley RA (2009) Climate change impacts on northern Australian rangeland livestock carrying capacity: a review of issues. The Rangeland Journal 31: 1-29.

McSherry M, Ritchie M (2013) Effects of grazing on grassland soil carbon: a global review. Global Change Biology 19: 1347-1357.

McCullagh P (1980) Regression models for ordinal data. Journal of the Royal Statistical Society: Series B (Methodological) 42: 109-127.

Mueller L, Behrendt A, Shepherd TG, Schindler U, Ball BC, Khudyaev S, Kaiser T, Dannouski R, Eulenstein F (2014) Simple field methods for measurement and evaluation of grassland quality. In: Mueller L, Saparov 
A, Lischeid G (eds) Novel measurement and assessment tools for monitoring and management of land and water resources in agricultural landscapes of Central Asia. Environmental Science and Engineering. Springer International Publishing. Switzerland. pp: 199- 222.

Novák J (2004) Evaluation of grassland quality. Ekológia 23: 127-143.

NRC (1994) Rangeland health. New methods to classify, inventory, and monitor rangelands. National Academy of Sciences. National Academy Press. Washington, D.C., USA. 180p.

Oba G (2012) Harnessing pastoralists' indigenous knowledge for rangeland management: three African case studies. Pastoralism: Research, Policy and Practice 2: 1-25.

Pellant M, Shaver P, Pyke D, Herrick JE (2005) Interpreting indicators of rangeland health. Version 4. Technical Reference 1734-6. USDI-BLM-NSTC. Denver, Col. USA. 122p.

Perkins LB, Blank RR, Ferguson SD, Johnson SW, Lindemann WC, Raue BM (2013) Quick start guide to soil methods for ecologists. Natural Resource Management Faculty Publications 101. http://openprairie.sdstate. edu/nrm_pubs/101. Fecha de consulta: 14 de abril de 2021.

Pyke DA, Herrick JE, Shaver P, Pellant M (2002) Rangeland health attributes and indicators for qualitative assessment. Journal of Range Management 55: 584-597.

Ricard MF, Viglizzo EF (2020) Improving carbon sequestration estimation through accounting carbon stored in grassland soil. MethodsX 2020: 100761. DOI: 0.1016/j.scitotenv.2019.01.130.

Sanaei A, Ali A, Chahouki MAZ, Jafari M (2018) Plant coverage is a potential ecological indicator for species diversity and aboveground biomass in semi-steppe rangelands. Ecological Indicators 93: 256-266.

Sayre NF, de Buys W, Bestelmeyer B, Havstad KM (2012) "The range problem" after a century of rangeland science: New research themes for altered landscapes. Rangeland Ecology \& Management 65:545-552

Shao H, Sun X, Wang H, Zhang X, Xiang Z, Tan R, Chen X, Xian W, Qi J (2016) A method to the impact assessment of the returning grazing land to grassland project on regional eco-environmental vulnerability. Environmental Impact Assessment Review 56: 155-167.

Sinclair SJ, Griffioen P, Duncan DH, Millett-Riley JE, White MD (2015) Quantifying ecosystem quality by modeling multi-attribute expert opinion. Ecological Applications 25: 1463-1477.

Sinclair SJ, Avirmed O, White MD, Batpurev K, Griffioen PA, Liu C, Jambal S, Sime H, Olson KA (2021) Rangeland condition assessment in the Gobi Desert: A quantitative approach that places stakeholder evaluations front and Centre. Ecological Economics 181: 106891. DOI: 10.1016/j.ecolecon.2020.106891.

Ter Braak CJ (1986) Canonical correspondence analysis: a new eigenvector technique for multivariate direct gradient analysis. Ecology 67: 1167-1179.

Tongway D, Hindley N (2004) Landscape function analysis: A system for monitoring rangeland function. African Journal of Range and Forage Science 21: 109-113.

USDA, NRCS (2003) National range and pasture handbook. Revised version. USDA, NRCS, Grazing Lands Technol. Inst. 190-vi-NRPH, Washington, D.C. https://directives.sc.egov.usda.gov/OpenNonWebContent. aspx?content=17734.wba. Fecha de consulta: 7 de septiembre de 2020.

Van der Westhuizen HC, Snyman HA, Fouché HJ (2005) A degradation gradient for the assessment of rangeland condition of a semi-arid sourveld in southern Africa. African Journal of Range and Forage Science 22: 47-58. 